\title{
Use of a Conical Axicon as a Surface Acoustic Wave Focusing Device
}

\author{
ABDULLAH ATALAR AND HAYRETTIN KÖYMEN
}

\begin{abstract}
Ultrasonic axicons generate waves which focus on a line. They are used in various imaging applications as bulk wave focusing devices with a very long depth of focus. A new type of conical axicon is introduced. It consists of a concave parabolic surface immersed in a liquid medium and insonified obliquely by wavefronts generated by a plane transducer. The parabolic cylinder can be approximated by a portion of a circular cylinder without losing significantly in the focusing performance of the axicon. It is also shown that conical axicons can be used to excite surface waves provided that the cone angle of the axicon coincides with the Rayleigh critical angle of the liquid-solid interface. The generated surface waves focus into a diffraction-limited spot. This new surface wave focusing scheme is easy to use and has a conversion efficiency and sensitivity far better than other existing techniques.
\end{abstract}

\section{INTRODUCTION}

QURFACE acoustic waves (SAW's) are increasingly Wused in determining the surface properties of materials, as acoustical nondestructive testing (NDT) develops into a quantitative science. The spatially confined nature of SAW's provides an effective means to investigate the surface and near-surface characteristics of the materials. The type of information conveyed by the use of existing techniques is generally limited to the detection of surface irregularities, such as cracks. On the other hand, obtaining the detailed information, required to characterize the irregularity rather than merely detecting it, calls for methods which provide better spatial resolution or has more sophisticated structure and signal processing techniques.

The problem of obtaining the most detailed information by means of methods based on SAW can be decomposed into two parts. First of all, the SAW must be efficiently generated on the surface of the material of interest, which is mostly nonpiezoelectric; and secondly, the generated SAW must be focused into the smallest possible area. The generation of SAW's on nonpiezoelectric materials has been studied and various techniques are proposed in the literature.

Electromagnetic generation of SAW's has been achieved through the use of EMAT's [1]. These transducers are practical to use, but they have very low conversion efficiencies [2]. Another method is to use a wedge SAW transducer, through which one can first generate a bulk acoustic wave and then convert it into a SAW di-

Manuscript received August 8, 1985; revised April 8, 1986.

The authors are with the Electrical and Electronic Engineering Department, Middle East Technical University, Ankara, Turkey.

IEEE Log Number 8611070 . rectly on the surface to be examined [3], [4]. The problems associated with the use of various solid and liquid wedge materials are extensively investigated on both theoretical and experimental bases. While solid wedge transducers provide mechanical rigidity and ease of operation, the liquid wedge systems maintain the critical advantage of high conversion efficiency [5]. When the bulk compressional waves are scattered by surface grooves, Rayleigh waves are strongly generated. This phenomenon of compressional to SAW conversion is employed as another means of SAW generation on nonpiezoelectric materials [6].

None of these generation methods, as they stand, are suitable for focusing SAW. Fitzpatrick et al. [7] devised a method to image surfaces using SAW. They use a tilted spherical bulk wave transducer to excite circularly divergent surface waves. These waves are detected by a second transducer after they leak into the liquid. In another application, Smith et al. [8] proposed a method of generating convergent SAW's on the surface to be examined, by defocusing a spherical acoustic microscope lens employing a semicircular disk transducer. The generated SAW focuses on a spot coinciding with the axis of the lens. The reflected SAW caused by surface irregularities will be received by the same transducer operating in the pulse-echo arrangement. They have shown that an acoustic image taken by a regular scanning acoustic microscope in defocused condition is indeed mostly a focused SAW image. The principal drawback in both of these SAW focusing systems is that the amount of energy converted into SAW is limited to the energy emitted from a very narrow strip on the surface of the lens, the width of which is determined by the spread of the Rayleigh critical angle.

Another method is proposed by Nongaillard et al. to overcome the disadvantages of previous systems. They use a cylindrical lens tilted at the Rayleigh critical angle to excite focused SAW [9]. A greater bulk-to-SAW conversion efficiency is obtained in this system, but still not all of the incident rays are at the Rayleigh critical angle and the SAW energy is spread over a line rather than being focused on a spot.

We introduce a novel method of focusing SAW's on the surface of nonpiezoelectric materials by using a new type of conical axicon. Axicon is the name commonly given to wave generators which focus the waves on a line. In this paper the axicons which produce waves with conical equal phase fronts will be referred to as "conical axi- 


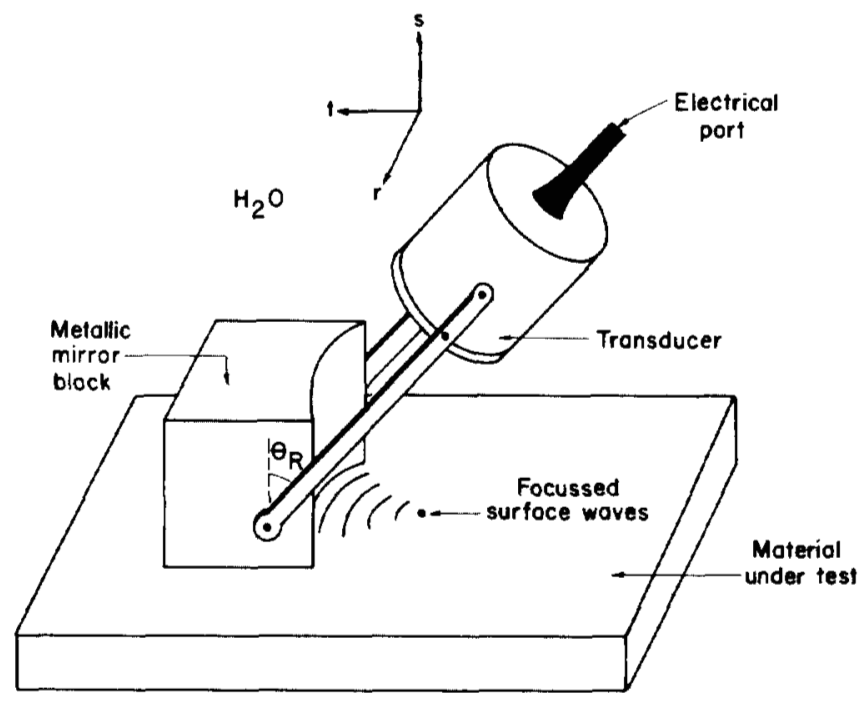

Fig. 1. Geometry to obtain focused surface wave.

cons." Axicons have been proposed as bulk acoustic wave imaging devices. A high spatial resolution can be achieved in the vicinity of their axis. This is a consequence of their large aperture. It can, however, be shown [10] that this large aperture yields a poor impulse response even for reflectors on the focal axis. When axicons are used in pulseecho mode as bulk wave focusing devices, the linearity of phase variation along the axis is particularly important. If the structure of the axicon does not provide this property, then the ultrasonic pulse is dispersed in range, and therefore range estimation is ambiguous. In conical axicons the phase variation along the axis is linear. Farnell et al. [11] and Kobayashi et al. [12] used axicons as planar lenses for low-frequency acoustic microscopes. On the other hand, axicons are used in medical imaging either alone [13] or in conjunction with spherical focusing transducers [14] with the advantage of having an extended depth of focus, despite their inherent off-axis sensitivity and the difficulty of manufacturing.

We will show in this paper that a conical axicon with the proper cone angle can be used to excite surface waves focusing into a diffraction limited spot. The technique allows one to scan the surface to be examined and thus to image the associated properties of materials. We propose an axicon that has a very simple construction and is easy to fabricate.

\section{Principle of Operation}

Consider the geometry shown in Fig. 1. An acoustic beam generated by a piston transducer in the liquid medium is obliquely incident on a concave parabolic cylinder surface of a solid. If the incidence angle is high enough, all of the incident power will be specularly reflected, no bulk waves will be excited in the solid medium, and the medium will act like a parabolic cylinder mirror.

Suppose that the incident beam is represented by a plane wave $\exp \left[j\left(k_{S} s+k_{T} t\right)\right]$. Upon reflection the $s$ or $t$ de- pendence will not change (except a sign change in $t$ ), but it will acquire an $r$ dependent term which takes care of the reflection at the parabolic surface. Recalling that a parabolic cylinder mirror focuses a normally incident plane wave into a line focus, an obliquely incident plane wave will be focused into a line with a linearly varying phase. A line focus was obtained earlier using axicons [15]-[17]. Note that the wavefronts in the arrangement of Fig. 1 are conical and the axis of the cone coincides with the focal line of the parabolic cylinder.

Now suppose that a material with a plane surface is placed perpendicular to this axis. The intersection of the conical wavefronts with the material surface is always circular. If the size of the parabolic cylinder is finite, the reflected wave will be a section of the conical surface, and hence the intersection with the material surface will be a circular arc rather than a complete circle.

It is well-known that a beam incident on a liquid-solid interface excites surface waves strongly, provided that the incidence angle is equal to the Rayleigh critical angle. We can combine this fact with our ultrasonic axicon to generate a focused surface wave. The incidence angle of the beam created by the transducer is adjusted to equal the Rayleigh critical angle. As the conical wavefront propagates towards the interface, the intersection with the material surface will be a circular arc with diminishing radius. The excited surface wave will reinforce the surface wavefront previously excited when the arc radius was larger. This is because of the fact that the incidence angle selected matches the $k$ vector components along the interface. Notice that by this process all the energy in a conical wavefront is converted into a single circularly converging wavefront of the surface wave.

The arrangement proposed by Nongaillard et al. [9] involves oblique cylindrical wavefronts and in this case, the intersection with the material surface would be elliptical instead of circular. These elliptical wavefronts are always of the same size, and they will not reinforce the previously excited wavefronts. Moreover, the incidence angle for the liquid-solid interface is not fixed at the Rayleigh angle throughout the elliptical intersection. Therefore, only a fraction of the incident bulk wave energy is converted into SAW, and the excited SAW is not focused on a spot but rather spread over a line. If the incident wavefront were a spherically converging wave [8], only a small fraction of the energy would be converted into a surface wave, since only a small section of the incoming wavefront would be incident at the Rayleigh critical angle.

Our conical wavefront will generate a diffraction-limited focal point, and as a result, focused surface waves are obtained with almost all the acoustic power created by the transducer. The impulse response of this focusing system is inherently very short. The deficiency in the impulse response of axicons does not play any role in this case.

The surface wave generated is only temporary; it will eventually leak back into the liquid medium as a bulk wave. The surface waves start leaking as soon as they are generated. If the material surface is perfectly smooth 
without any obstructions (i.e., no surface wave reflectors), almost none of the incident power will return to the transducer. We note that excitation of a backward traveling surface wave is possible [18], [19] because the incident beamwidth is finite and its angular spectrum contains a plane wave which is able to excite the backward traveling surface wave. This wave will cause some transducer output when it leaks into the liquid medium, so there will be a transducer output even though no surface wave reflectors are present. However, this effect is very small, and it can be further reduced by expanding the beamwidth and/or suitably shaping it. The transducer will receive an appreciable power only if a surface wave reflector is present which changes the propagation direction of the forward traveling surface waves. Suppose that a reflector exists at focal spot of the surface waves. In this case the reflected surface waves will be circularly divergent. They leak into the liquid medium to reconstruct the conical wavefront which originally created the surface waves. The conical wavefront will get collimated in the transducer direction after being reflected from the parabolic mirror. The transducer will, therefore, receive an acoustic power because of the presence of the reflector at the surface wave focus. If the reflector is not exactly at the surface wave focus, the reflected wavefront will not be an exact replica of the incident wavefront, and hence the transducer output voltage will be reduced.

\section{THEORY}

To be able to analyze the geometry described in the previous section, we will first treat a liquid wedge transducer geometry. Various treatments of wedge transducers were given in the literature [4], [5]. Nevertheless, we include a brief derivation for the sake of completeness. Then we will concentrate on the focused surface wave case using the parabolic mirror. The SAW field at the focus will be investigated to determine the resolution behavior of the system. The optimal size of the transducer is also determined to help in designing SAW focusing systems.

\section{Wedge Transducer}

Suppose that a collimated acoustic beam is incident on the liquid-solid interface from the liquid side at the Rayleigh critical angle $\theta_{R}$, as shown in Fig. 2. All of the incident waves will be converted into surface waves, but the surface waves will start to leak back to the liquid medium as soon as they are generated.

The leak field $p_{L}(z)$ along the interface $(z$ axis) can be calculated by [20]

$$
p_{L}(z)=-2 \alpha_{L} \int_{-\infty}^{z} p_{I}\left(z^{\prime}\right) \exp \left[j k_{P}\left(z-z^{\prime}\right)\right] d z^{\prime}
$$

where $p_{I}(z)$ is the incident field along the interface, $k_{P}=$ $k_{R}+j\left(\alpha_{D}+\alpha_{L}\right)$, and $\alpha_{D}$ and $\alpha_{L}$ are the dissipation and leak rates of the surface wave.

We assume that there is an incident field only for $-f<z<0$ (see Fig. 3.). In this case we write the incident field as

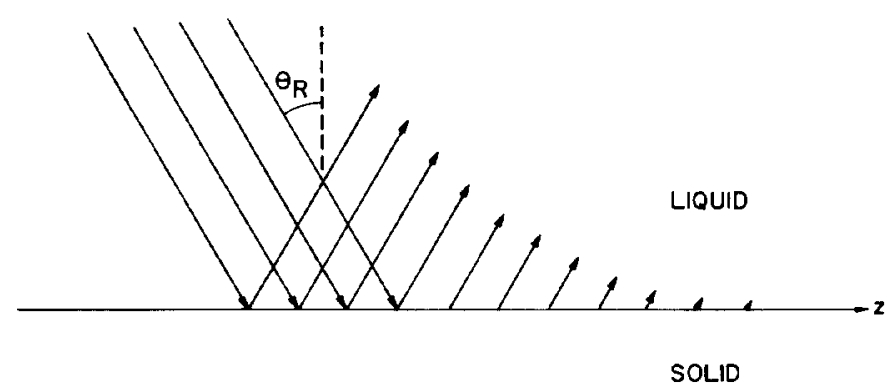

Fig. 2. Collimated acoustic beam is incident on liquid-solid interface at Rayleigh critical angle.

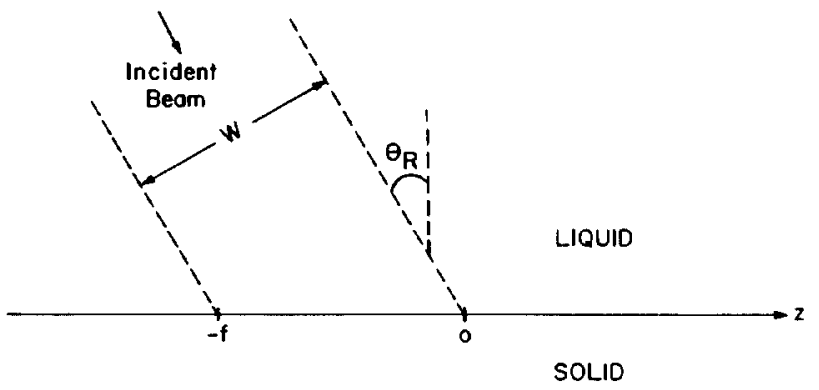

Fig. 3. Geometry of Rayleigh wave excitation by bounded beam in liquid wedge.

$$
p_{I}(z)= \begin{cases}\exp \left[j k_{R} z\right], & -f<z<0 \\ 0, & \text { otherwise. }\end{cases}
$$

Substituting (2) in (1) results in

$$
\begin{aligned}
p_{L}(z)= & -2 \alpha_{L} \int_{-f}^{0} \exp \left[j k_{P} z\right] \exp \left[\left(\alpha_{D}+\alpha_{L}\right) z^{\prime}\right] d z^{\prime} \\
= & \frac{-2 \alpha_{L}}{\alpha_{D}+\alpha_{L}} \exp \left[j k_{R} z\right] \exp \left[-\left(\alpha_{D}+\alpha_{L}\right) z\right] \\
& \cdot\left\{1-\exp \left[-\left(\alpha_{D}+\alpha_{L}\right) f\right]\right\}, \quad \text { for } z>0 .
\end{aligned}
$$

Now suppose that a surface wave reflector exists at $z=$ 0 with a reflection coefficient $R$. In this case the reflected leak field $p_{R L}(z)$ for $z<0$ can be written as

$$
\begin{aligned}
p_{R L}(z)= & \frac{-2 \alpha_{L}}{\alpha_{D}+\alpha_{L}} R \exp \left[-j k_{R} z\right] \\
& \cdot \exp \left[\left(\alpha_{D}+\alpha_{L}\right) z\right]\left\{1-\exp \left[-\left(\alpha_{D}+\alpha_{L}\right) f\right]\right\}
\end{aligned}
$$

which is found by substituting $-z$ in place of $z$ in (3).

The reflected leak field will propagate just in the reverse direction of the original beam. If this leak field is to be received by the same transducer generating the incident beam, the output voltage $V_{R}$ of the transducer can be expressed approximately as [21]

$$
V_{R}=\int_{-\infty}^{+\infty} p_{R L}(z) p_{I}(z) d z
$$

Using (2) in (5) we find 


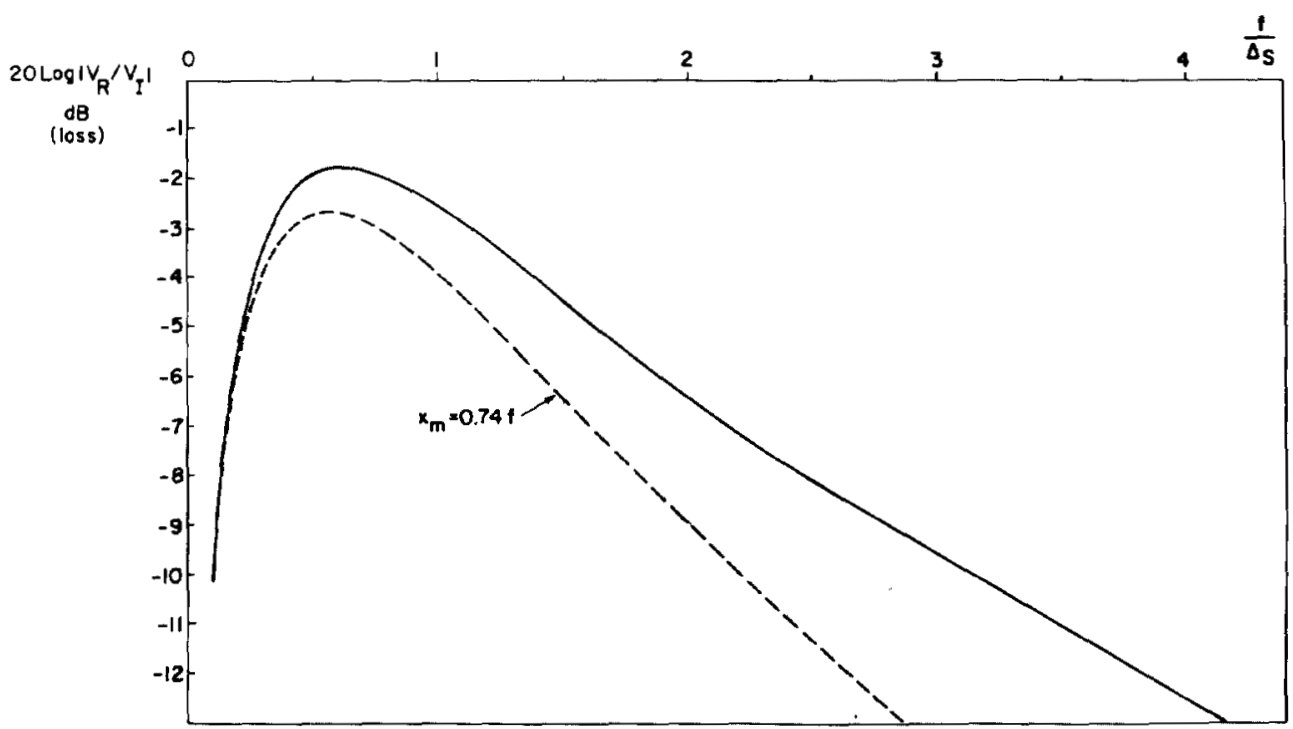

Fig. 4. Normalized output voltage (20 $\left.\log \left|V_{R} / V_{l}\right|\right)$ as function of $f / \Delta_{S}$. Solid line is for wedge transducer and broken line is for focused case.

$$
V_{R}=\int_{-f}^{0} p_{R L}(z) \exp \left[j k_{R} z\right] d z
$$

Equation (6) can be simplified to read

$$
V_{R}=\frac{-2 \alpha_{L}}{\left(\alpha_{D}+\alpha_{L}\right)^{2}} R\left\{1-\exp \left[-\left(\alpha_{D}+\alpha_{L}\right) f\right]\right\}^{2} .
$$

To optimize the parameters of the system, we have to compare this value to a reference. Let $V_{l}$ represent the output voltage of the transducer for the case where the incident beam is reflected back into the transducer by a planar perfect reflector surface placed normal to the beam axis. Using (5) one finds $V_{I}=f$. Therefore, we can write

$$
\frac{V_{R}}{V_{I}}=\frac{-2 \alpha_{L}}{f\left(\alpha_{D}+\alpha_{L}\right)^{2}} R\left\{1-\exp \left[-\left(\alpha_{D}+\alpha_{L}\right) f\right]\right\}^{2} .
$$

To find the optimum beamwidth $W$ which will maximize $V_{R} / V_{l}$, we differentiate (8) with respect to $f$ and equate it to zero to find

$$
f=1.2564 /\left(\alpha_{D}+\alpha_{L}\right) \text { and } W=f \cos \theta_{R} .
$$

For this beamwidth we get the maximum transducer output

$$
\left|V_{R} / V_{l}\right|_{\max }=0.8145 R /\left(\alpha_{D}+\alpha_{L}\right) .
$$

As an example, assume that $\alpha_{D}=0$ and that $\alpha_{L}=$ $2 / \Delta_{S}$ where $\Delta_{S}$ is the Schoch displacement [22]. For this case we find

$$
f / \Delta_{S}=0.6282 \text { or } W / \Delta_{S}=0.6282 \cos \theta_{R}
$$

and

$$
\left|V_{R} / V_{l}\right|_{\max }=0.8145 R \text {. }
$$

For the optimum beamwidth, the loss incurred by the system is only $1.78 \mathrm{~dB}$, not counting the reflection loss. This surprisingly low value of loss is an inherent property of wedge transducers, and it is due to surface wave com- ponents that leak into liquid medium before they reach the reflector, and also due to the reflected surface wave components that stay as a surface wave too long to be collected by the wedge transducer.

In Fig. 4, $20 \log \left|V_{R} / V_{I}\right|$ is plotted (solid line) as a function of $f / \Delta_{S}$. Observe that the variation of the curve around the maximum is rather slow, suggesting that the beamwidth is not an extremely critical parameter to get minimum loss in the system.

\section{SAW Focusing Using an Axicon}

Now let us analyze the problem involving a parabolic cylinder reflector placed vertically on the interface between the liquid and the solid. Suppose the parabola has a focal distance of $f$ and it extends from $-x_{m}$ to $x_{m}$ as shown in Fig. 5. The beamwidth $W$ is selected so as to illuminate the liquid-solid interface between the reflector edge and the focal point. Mluminating the interface beyond the focal point is useless because that part can only give rise to diverging surface waves. The height of the reflector is chosen to match the beamwidth which is equal to $f \cot \theta_{R}$.

The uppermost (minimum $y$ position) ray hitting the mirror surface at $x=0$ will penetrate into the solid medium just at the focal point $f$, as shown in Fig. 6. However, the uppermost ray at $x=x^{\prime}$ will not reach as far as the focal point when it penetrates into the solid medium. For this ray, (3) must be changed to

$$
\begin{aligned}
-2 \alpha_{L} & \int_{-d-f}^{-d} \exp \left[j k_{P} z\right] \exp \left[\left(\alpha_{D}+\alpha_{L}\right) z^{\prime}\right] d z^{\prime} \\
= & \frac{-2 \alpha_{L}}{\alpha_{D}+\alpha_{L}} \exp \left[j k_{R} z\right] \exp \left[-\left(\alpha_{D}+\alpha_{L}\right) z\right] \\
& \cdot\left\{\exp \left[-\left(\alpha_{D}+\alpha_{L}\right) d\right]-\exp \left[-\left(\alpha_{D}+\alpha_{L}\right)(d+f)\right]\right\} .
\end{aligned}
$$

To find the total voltage, we modify (4) appropriately, we integrate over $x$ with $d=\left(\frac{1}{4} f\right) x^{2}$ and get 


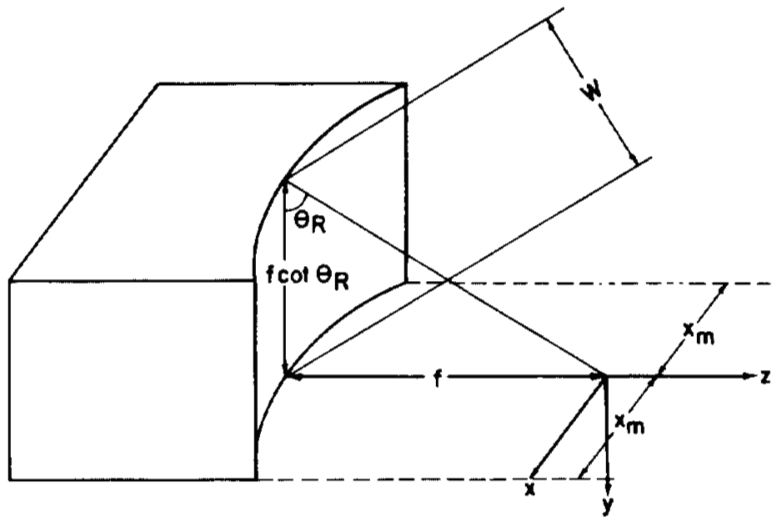

Fig. 5. Geometry of parabolic mirror.

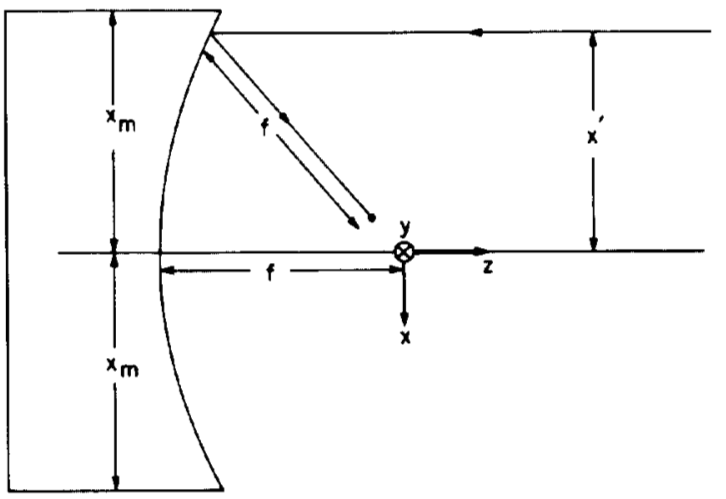

Fig. 6. Positions of different rays reflecting from parabolic mirror.

$$
\begin{aligned}
\left|\frac{V_{R}}{V_{l}}\right|= & \frac{2 R \alpha_{L}}{f x_{m}\left(\alpha_{D}+\alpha_{L}\right)^{2}}\left\{1-\exp \left[-\left(\alpha_{D}+\alpha_{L}\right) f\right]\right\}^{2} \\
& \cdot \int_{0}^{x_{m}} \exp \left[-\left(\alpha_{D}+\alpha_{L}\right) x^{2} / 2 f\right] d x .
\end{aligned}
$$

Note that the reflection coefficient is assumed independent of angle of incidence and is equal to $R$.

Recalling that [23]

$$
\int_{0}^{x} \exp \left[-t^{2} / 2\right] d t=(\pi / 2)^{1 / 2} \operatorname{erf}\left(x / 2^{1 / 2}\right)
$$

we arrive at

$$
P(r, s)=\left\{\begin{array}{l}
\left\{\exp \left[-\left(\alpha_{D}+\alpha_{L}\right)\left(f-s \tan \theta_{R}\right)\right]\right. \\
0
\end{array}\right.
$$$$
\begin{aligned}
\left|\frac{V_{R}}{V_{l}}\right|= & \frac{2 R(\pi / 2)^{1 / 2} \alpha_{L}}{f x_{m}\left(\alpha_{D}+\alpha_{L}\right)^{2}}\left\{1-\exp \left[-\left(\alpha_{D}+\alpha_{L}\right) f\right]\right\}^{2} \\
& \cdot\left[f /\left(\alpha_{D}+\alpha_{L}\right)\right]^{1 / 2} \operatorname{erf}\left\{x_{m} /\left[2 f /\left(\alpha_{D}+\alpha_{L}\right)\right]^{1 / 2}\right\} .
\end{aligned}
$$

This function is plotted in Fig. 4 (broken line) as a function of $f / \Delta_{S}$ for the special case $\alpha_{D}=0, \alpha_{L}=2 / \Delta_{S}$, and $x_{m}=0.74 f$. The maximum is reached when $f / \Delta_{S}=0.55$, corresponding to a loss of $2.7 \mathrm{~dB}$. More loss occurs in this case compared to the wedge transducer case, and the optimal beamwidth is seen to have decreased slightly. One

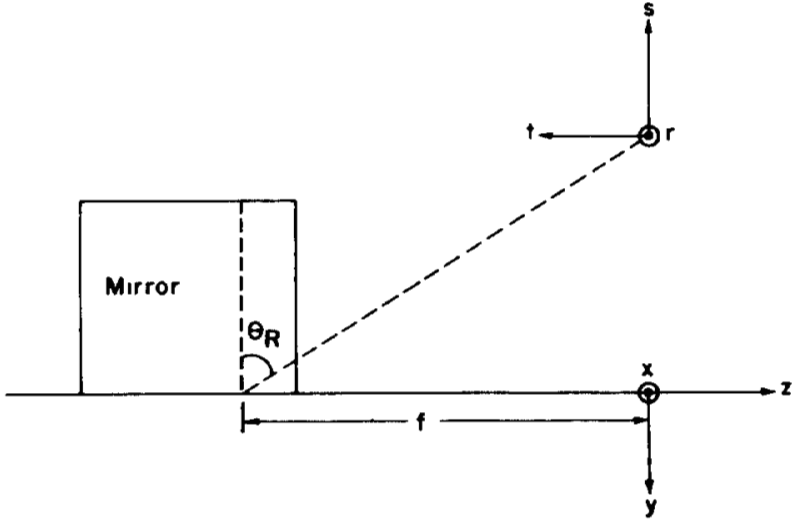

Fig. 7. Geometry for focal field calculation.

may expect that as $x_{m}$ is increased, the loss in the system will increase and the optimum beamwidth will be even smaller.

\section{Surface Wave Field at the Focus}

To find the surface wave field at the focus of the system, we consider the geometry drawn in Fig. 7. The bulk wave incident at the $r-s$ plane is represented by $u^{+}(r, s)$. The surface wave field along the $x$ axis and propagating in the $+z$ direction, $u_{f}^{+}(x)$, can be written as the Fourier transform of the integrated incident field, because the cylindrical parabolic mirror acts like a Fourier transform operator in one dimension [24] and the bulk-to-surface wave conversion phenomenon as an integrator in the other dimension:

$$
\begin{aligned}
u_{f}^{+}(x)= & \int_{-\infty}^{+\infty}\left\{\int_{-\infty}^{+\infty} u^{+}(r, s) P(r, s) d s\right\} \\
& \cdot \exp \left[-j\left(k_{R} / f\right) r x\right] d r
\end{aligned}
$$

where $k_{R}$ is real part of the wavenumber of surface waves and $P(r, s)$ is the pupil function due to the finite size of the parabolic mirror as obtained from a projection of mirror surface onto the $r-s$ plane. The pupil function should also include phase factors arising from the angle of incidence. $P(r, s)$ may be given by

$$
\begin{array}{ll}
\left.\exp \left[-j k_{R} s\right]\right\}, & \quad \text { for }-x_{m}<r<x_{m} \text { and } \\
& -r^{2} \cot \theta_{R} / 4 f<s<\left(f-r^{2} / 4 f\right) \cot \theta_{R} \\
& \text { otherwise. }
\end{array}
$$

The pupil function here is found assuming a mirror with rectangular cross section, a width of $2 x_{m}$, and a height of $f \cot \theta_{R}$. Equation (10), along with (11), can be used to determine the distribution of surface wave field at the focal plane. This field determines the resolution performance of the system. A tight field distribution results in a good resolution. An evaluation of the integral in (10) requires the knowledge of the field distribution at the back focal plane of the mirror $u^{+}(r, s)$. To be able to calculate the focal field distribution, we assume a Gaussian distributed incident field in $r-s$ plane: 


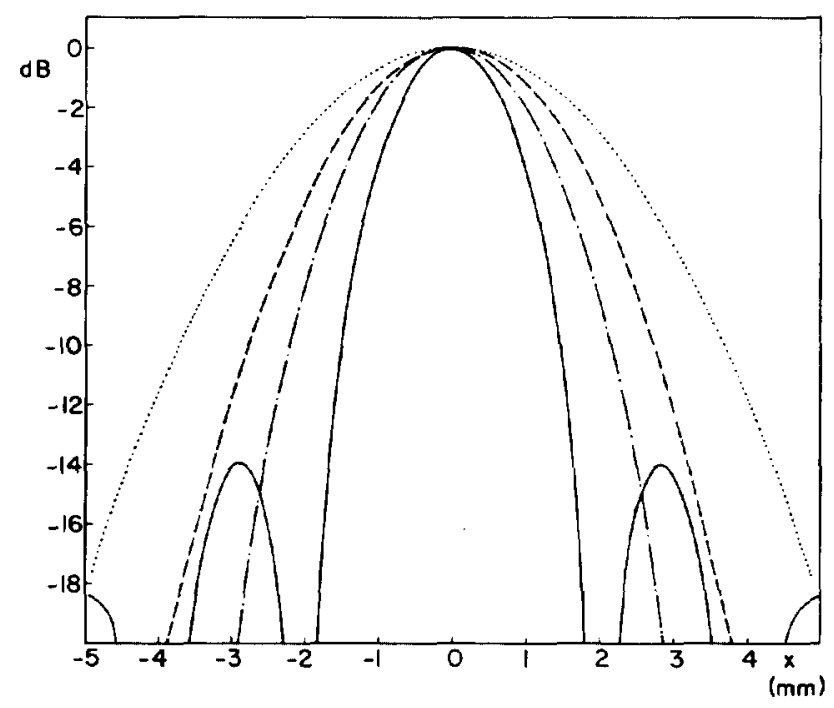

Fig. 8. Focal field distributions for various $W$. Solid line for $W=\infty$, dotdashed line for $W=2 x_{m}$, dashed line for $W=x_{m}$, and dotted line for $W$ $=x_{m} / 2$.

$$
u^{+}(r, s)=\exp \left[-\left(r^{2}+s^{2} \sin ^{2} \theta_{R}\right) / W^{2}\right] \exp \left[j k_{R} s\right]
$$

where $W$ is the transverse distance to the point where the beam's amplitude has been reduced to $1 / e$ of its maximum amplitude.

Equation (10) is evaluated numerically using the FFT algorithm in a Pascal program, and the results are plotted in Fig. 8 for $f=25 \mathrm{~mm}, x_{m}=18.5 \mathrm{~mm}, \theta_{R}=30^{\circ}$, $\lambda_{R}=3 \mathrm{~mm}$, and for various values of $W$. It is clear from the investigation of this plot that as $W$ gets smaller, the beamwidth at the focal plane increases. This should be expected from the Fourier transform relation between the field distributions at the two planes. The sidelobes are maximum when the $u^{+}(r, s)$ is a uniform plane wave ( $W$ is infinity). In this worst case, the sidelobes are $14 \mathrm{~dB}$ below the peak, and hence they are lower than the sidelobes of a $(\sin x) / x$ function. The reason for that is the pupil function $P(r, s)$ giving an apodization which reduces the sidelobes. $14 \mathrm{~dB}$ is quite a good value when this beam is used for imaging purposes. The sidelobe level can be further reduced by decreasing $W$. However, that will cause a broadening of the beam and thus a loss in resolution.

\section{Size of the Transducer}

The size of the transducer must be selected to give the desired illumination at the $r-s$ plane. A transducer much larger than the mirror size will create a relatively uniform illumination, and hence it will give a good resolution performance as discussed earlier, but a lot of acoustic power will be wasted since they miss the mirror surface. On the other hand, the size of the transducer can be selected to illuminate only the mirror surface without wasting any power; however, this selection may cause a degradation in the resolution. Therefore, a compromise has to be made between the high signal level and the good resolution performance.

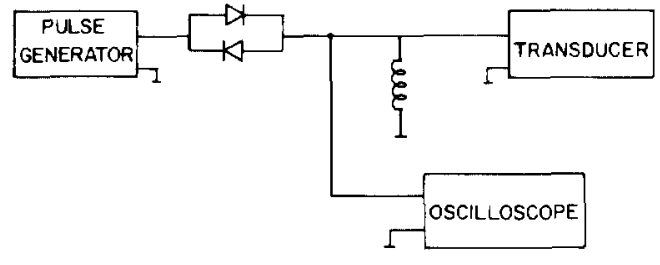

Fig. 9. Measurement setup.

The reasoning given is valid for a noise-limited system. However, the dynamic range of the system may be limited by the presence of a background signal level as discussed before. In this case, a lower bound on the size of the transducer must be specified in order not to excite plane waves at angles too far away from the Rayleigh critical angle $\theta_{R}$.

\section{EXPERIMENTAL WORK}

The experimental apparatus consists of a transducer, a cylindrical mirror, and other mechanical interconnections which allow the adjustment of the position and the inclination of the transducer with respect to the mirror (Fig. 1). The apparatus is immersed into water entirely for operation. The mirror is placed on the test piece such that the axis of the mirror is perpendicular to the surface of the test piece. The ultrasonic beam produced by the transducer is incident upon the mirror at an angle equal to 90$\theta_{R}$. Pulsed ultrasonic waves are transmitted by the transducer which are reflected by the mirror to form a conical phase front approaching the surface under test. The reflected waves are detected by the same transducer and the corresponding electrical signal is displayed on an oscilloscope. The electrical setup is schematically shown in Fig. 9. The apparatus is mounted on a micropositioning system, which allows the surface of the test piece to be scanned laterally.

\section{Transducer}

The transducer is a composite one which has a 1-in diameter PZT-4 ceramic disk, resonating at $1 \mathrm{MHz}$. The ceramic is matched to water load through a quarter-wavelength thick epoxy resin matching layer and backed by a highly attenuating and heavy backing made up of approximately $0.8-\mathrm{mm}$ diameter lead balls in an epoxy resin matrix. The backing provides the necessary damping to the resonating ceramic required for short pulse operation while reducing the sensitivity. The matching layer, on the other hand, helps to improve the sensitivity of the transducer by increasing the load on the front face which in turn provides further damping to the ultrasonic pulse.

The transducer is driven by an approximately $0.2-\mu \mathrm{s}-$ long voltage pulse which produces a decaying sinusoidal pressure pulse. The transmitted ultrasonic pulse is about $5 \mu$ s long and has a nominal center frequency of $1 \mathrm{MHz}$.

\section{Mirror}

To obtain a conical phase front, a mirror with parabolic concavity is required as explained earlier. However, such a mirror is difficult to machine. Instead, we designed a 
right cylindrical mirror as an approximation. The design criterion is such that when the mirror is insonified by a plane wave, the limiting ray reflected from the edge of the mirror is allowed to be out of phase with the central ray by not more than a quarter wavelength. Then the maximum width of the mirror, which does not produce any significant cylindrical aberration, is found as

$$
x_{m}(\max )=\left\{f \lambda_{R} / 2+4 f\left(f \lambda_{R} / 2\right)^{1 / 2}\right\}^{1 / 2}
$$

to a very good approximation, where $x_{m}$ is the half of the mirror width, $\lambda_{R}$ is the wavelength of the surface waves, and $f$ is the focal length of the mirror which is equal to the half of the radius of the cylinder.

The mirror used in this investigation is made by cutting a concave cylindrical surface into a block of brass. The height of the mirror is $38 \mathrm{~mm}$, its width is $37 \mathrm{~mm}$, the radius of the cylinder is $50 \mathrm{~mm}$, and thus the focal length of the mirror $f$ is $25 \mathrm{~mm}$. This width of the mirror is slightly less than the allowable maximum with the foregoing criterion and subtends an angle of $82^{\circ}$ at the focus. The $25-\mathrm{mm}$ focal length is somewhat larger than the optimum value for aluminum at $1 \mathrm{MHz}$, which is $17.5 \mathrm{~mm}$. However, Fig. 4 indicates that this nonoptimum focal length causes only $1-\mathrm{dB}$ extra insertion loss as compared to the optimum.

We used aluminum test pieces for all measurements. The Rayleigh wavelength at $1 \mathrm{MHz}$ is $2.85 \mathrm{~mm}$ in aluminum which determines the size of the diffraction-limited focus and the skin thickness of the excited leaky surface waves [3]. The thickness of the test pieces is chosen to be $10 \mathrm{~mm}$ so that the aluminum medium appears as a semi-infinite medium for all practical purposes.

When the apparatus is placed at the center of a sufficiently large test piece and properly adjusted for Rayleigh wave generation, only a single echo pulse can be observed on the oscilloscope (Fig. 10). This spurious echo is a consequence of the fact that the generated ultrasonic wave is not a perfect plane wave, but a bounded beam. The plane wave components of the beam propagating at angles far from $\theta_{R}$ are specularly reflected at the corner formed by the test piece and the mirror back to the transducer.

When the apparatus is moved towards the edge of the test piece such that the converging surface waves are focused on the edge, a significantly larger echo is produced about $17 \mu$ s after the first one (Fig. 11). The time required for acoustic waves to travel the 50-mm (twice the focal length) path at the Rayleigh velocity is $17 \mu \mathrm{s}$. Thus it is a very simple procedure to set the incidence angle accurately at $\theta_{R}$ : the mirror is placed a focal length $(25 \mathrm{~mm})$ away from the edge of the test piece, and the angle of inclination of the transducer is adjusted until the amplitude of this second echo arising from the edge reflection is maximized.

We have done various measurements to assess the performance of the focusing system. The lateral size of the focus is measured on an aluminum plate which has a vertical hole of $1-\mathrm{mm}$ diameter (Fig. 12). The mirror is placed a focal distance away from the hole so that the (a)

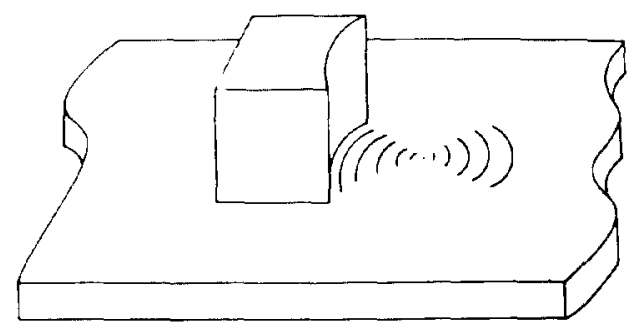

(b)
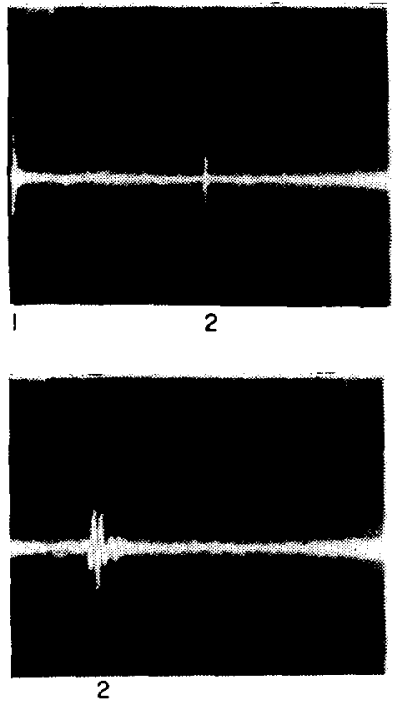

Fig. 10. Received echo pulses when edge of test plate is far away from focus. (a) Mirror geometry, (b) Oscilloscope trace of input pulse (1) and spurious pulse (2). (c) Expanded view showing just pulse (2).

(a)

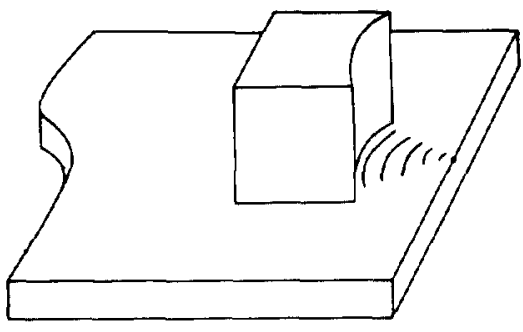

(b)
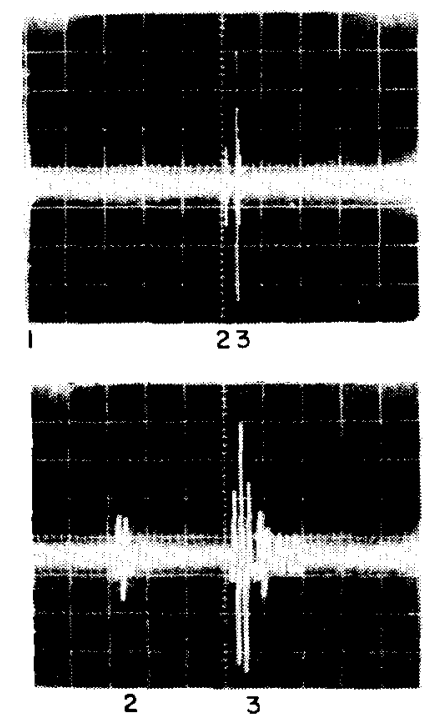

(c)

Fig. 11. Received echo pulses when edge of test plate is placed at focus (a) Mirror geometry. (b) Oscilloscope trace of input pulse (1), spurious pulse (2), and echo pulse from the focus (3). (c) Expanded view showing just pulses (2) and (3). 


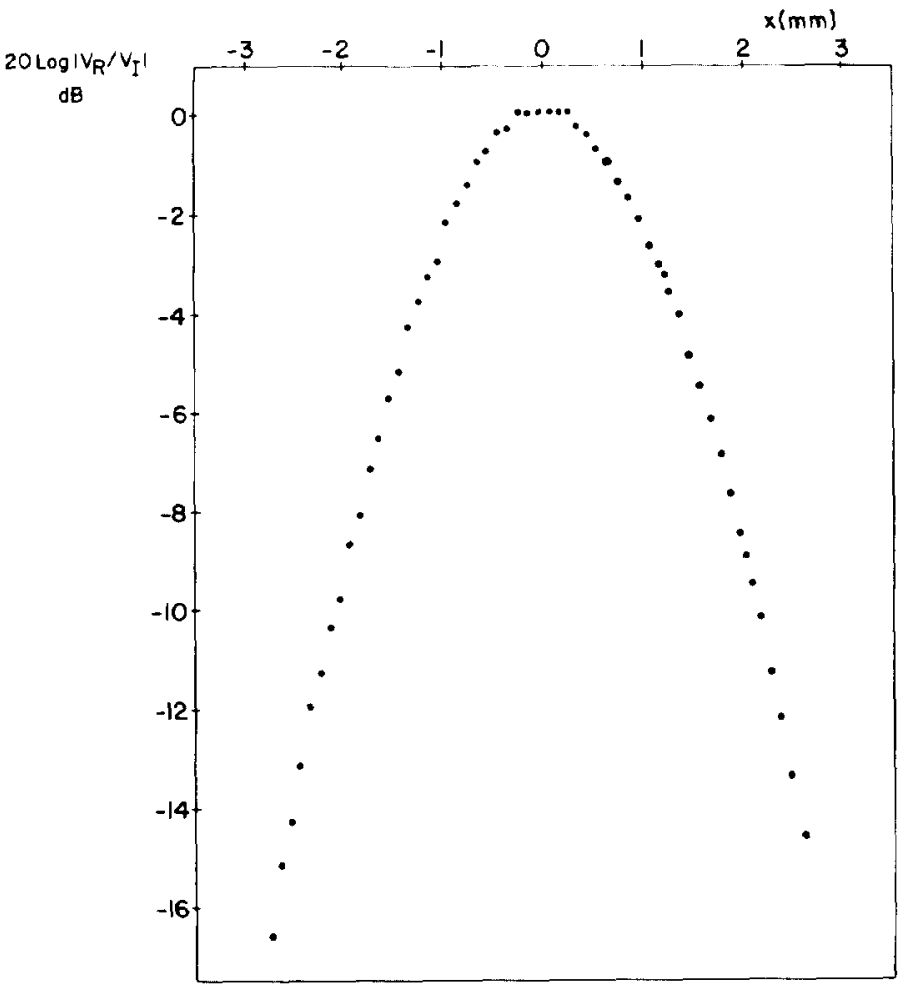

Fig. 12. Normalized output voltage $\left(20 \log \left|V_{R} / V_{l}\right|\right)$ as function of lateral displacement of 1 -mm diameter hole.

reflected Rayleigh wave amplitude is maximum. This reflected pulse amplitude is recorded as the mirror is moved laterally along the $x$ axis. The variation is shown in Fig. 12 . It can be observed that in a $2.2-\mathrm{mm}$ lateral displacement the reflected amplitude is within $3 \mathrm{~dB}$ of the maximum. This measurement compares well with Fig. 8 for the case when $W=x_{m}$. Note that the square of the field solution (doubling of $\mathrm{dB}$ scale) should be compared with the measurement to include two-way propagation.

We have measured the insertion loss of the surface wave conversion and reflection process to be able to compare with our theoretical predictions. First, the signal level $\left(V_{R}\right)$ is measured when the focal point of the focusing system coincides with the $90^{\circ}$ edge of a thick aluminum plate. Then the aluminum is placed directly against the transducer at a distance equal to the separation between the transducer and the mirror in the focusing system. The angle of the surface of the plate is adjusted to be perpendicular to the beam axis to get the maximum transducer output $\left(V_{l}\right)$. The ratios of two measurements is found to be $V_{R} / V_{I}=0.32$. We need a correction here to make a direct comparison with (9): (9) specifies $V_{I}$ for a perfect reflector, but in the experiment aluminum is used with finite reflection loss. When the reflection coefficient of the water-aluminum interface at normal incidence is taken into account we get

$$
V_{R} / V_{l}=0.27 \text { or }-11.4 \quad \mathrm{~dB} .
$$

A set of measurements are performed to find the lateral spatial resolution. Test pieces which contain lines of 1$\mathrm{mm}$ diameter holes are machined for this purpose (Fig.

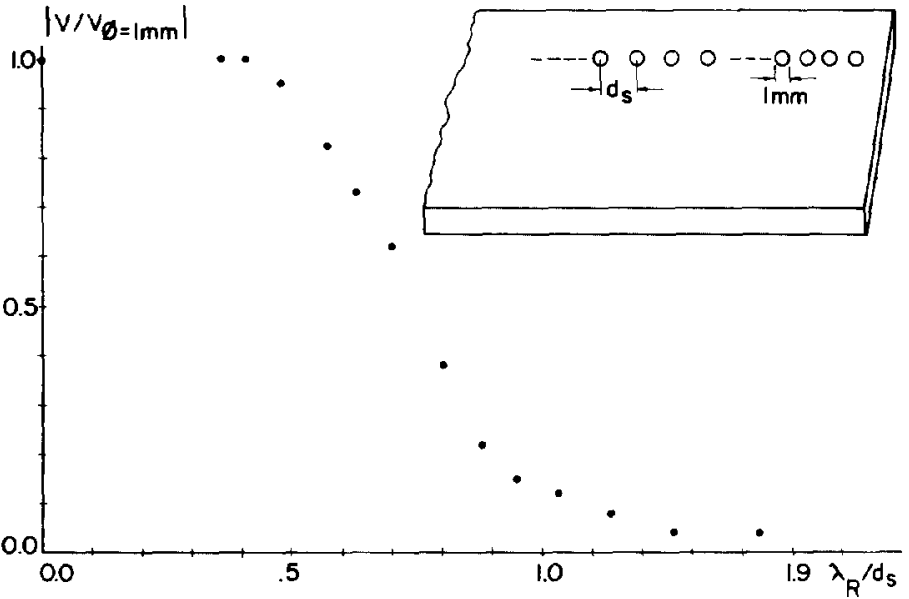

Fig. 13. Modulation transfer function for focused SAW beam as measured by series of holes with varying spacings.

13). Each line consists of ten equally spaced holes. Fifteen such lines of holes are drilled with different hole spacings ranging from 2 to $10 \mathrm{~mm}$. The system is focused on the line of holes, which is lying along the $x$ axis. The variation of the peak of the reflected signal amplitude (PRA) is recorded as the mirror is laterally moved along the $x$ axis. For every line with different hole spacing, the peak-to-peak variation of the PRA is calculated and plotted against the hole spacing in Fig. 13. The vertical axis is normalized to the peak value of the echo from a single $1-\mathrm{mm}$ hole reflector, while the horizontal axis is the ratio of the Rayleigh wavelength at $1 \mathrm{MHz}$ to the spacing of the holes $d_{S}$. It is observed that when $d_{S}$ is greater than 7 $\mathrm{mm}$, every hole in the line is well isolated. The variation in PRA is, therefore, exactly the same as that for a single hole. The peak-to-peak PRA variation falls off as $d_{S}$ gets smaller. The 3-dB point is reached at approximately $d_{S}=$ $4.5 \mathrm{~mm}$. This value of $d_{S}$ is about twice the size of the diffraction limited focus, $2.2 \mathrm{~mm}$.

We tested the sensitivity of the system to the size of the surface flaws by approximating the flaw by a hole drilled perpendicular to surface. A test piece is machined such that it contains spatially isolated holes of diameters ranging from 0.35 to $10 \mathrm{~mm}$. The peak amplitude of the echo from the edge of the hole is recorded when this edge is at the focus. This peak value is normalized to the vertical straight edge echo amplitude and plotted in Fig. 14 against the hole diameter normalized to the Rayleigh wavelength. It is observed that the reflection from the edge of $10-\mathrm{mm}$ diameter hole is almost as large as the reflection from the straight edge. The echo amplitude steadily falls as the hole diameter gets smaller, down to $-18.5 \mathrm{~dB}$ at $0.35 \mathrm{~mm}$ diameter.

The last set of measurements was made to get an understanding of the sensitivity of the focused Rayleigh waves to subsurface flaws. A special test piece is fabricated for this investigation, which contains holes with 1.5$\mathrm{mm}$ diameter and drilled from the back face of the plate (Fig. 15). The holes are drilled such that they are well separated and they have flat bottom. Their depth is ad- 


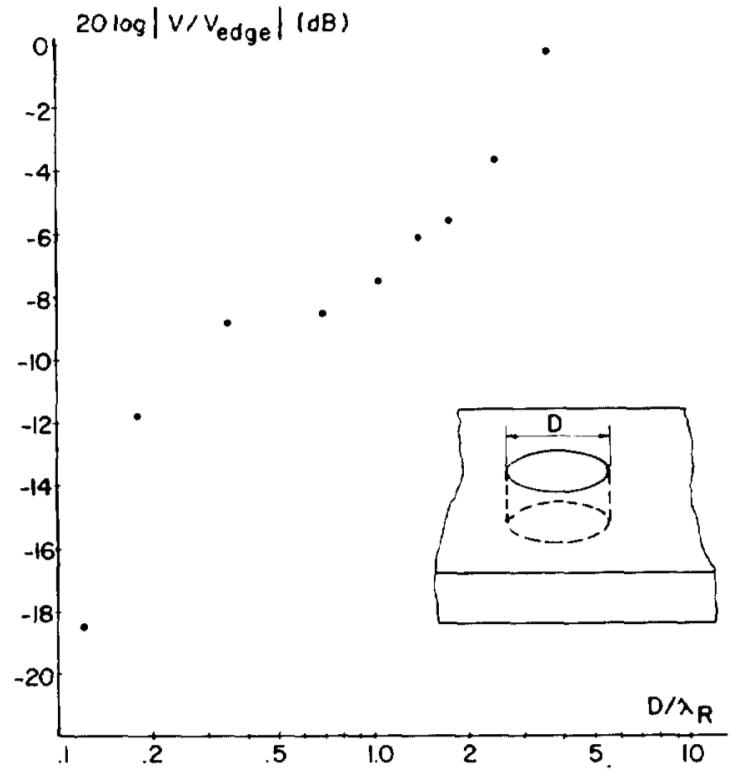

Fig. 14. Normalized output voltage as function of normalized hole diameter.

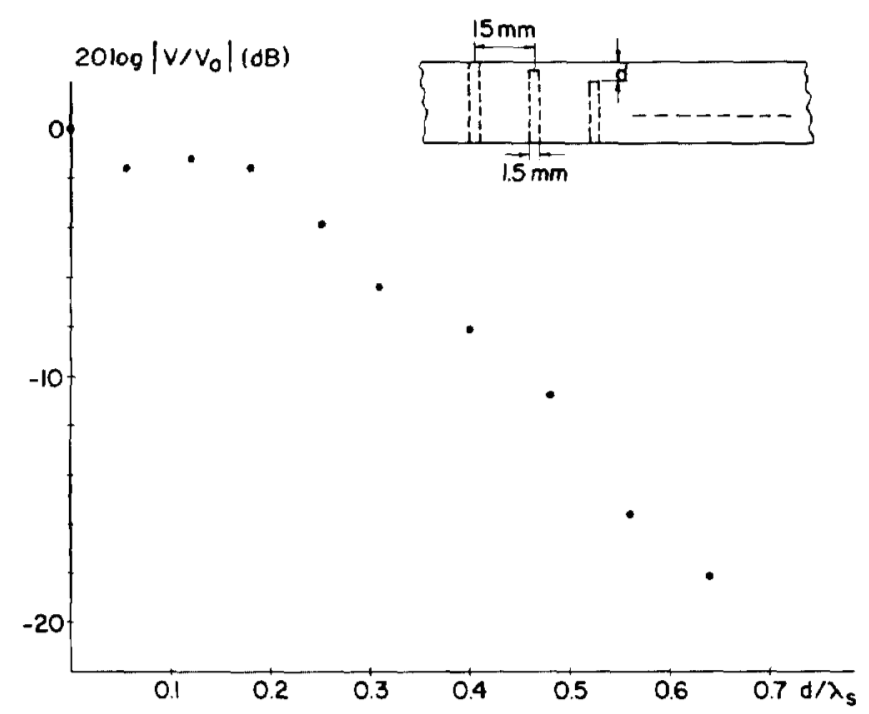

Fig. 15. Normalized output voltage as function of normalized inhomogeneity depth.

justed so that there is a different distance $d$ between the surface and the bottom of each hole. Thus 14 subsurface holes with different depths $d$ ranging from 0 to $3.1 \mathrm{~mm}$ are obtained. The mirror is focused on each hole, and the peak amplitude of the reflected echo is recorded. This peak value is normalized to that of the first one for which $d=$ 0 . The results are shown in Fig. 15 where the horizontal axis is the subsurface depth normalized with respect to the shear wavelength $\lambda_{s}$. It can be observed that the echo amplitude falls quite regularly as $d$ increases. The $3-\mathrm{dB}$ point is reached at about $d=0.9 \mathrm{~mm}$. As $d$ increases to more than 0.65 of $\lambda_{s}$, the normalized respective echo amplitude falls to less than 0.1 , and its level is about the same as the background signal. Note that the size of the detected inhomogeneity is only half as large as the SAW wavelength.
As mentioned earlier, an inherent background signal exists in the system. We monitored this background signal level on a large piece of aluminum plate and compared it to the peak amplitude of the echo from an edge; we observed that the dynamic range of this system is approximately $35 \mathrm{~dB}$.

\section{Discussion}

Realization of the surface wave focusing axicon has provided the expected very high signal level. The fact that the incident power is converted almost completely into a surface wave provides this favorable result. However, a noise-limited dynamic range is not possible due to the excitation of backward travelling surface waves. These waves cause a signal dependent background. To maximize the dynamic range, the beam generated by the transducer should be properly shaped to prevent the excitation of unwanted surface waves.

The insertion loss of the system used in experiments can be calculated using the theoretical results. At $1 \mathrm{MHz}$ the surface wavelength in aluminum is $2.85 \mathrm{~mm}$, and the corresponding Schoch displacement is $30.8 \mathrm{~mm}$. With a mirror of focal length $f=25 \mathrm{~mm}$ and $x_{m}=18.5 \mathrm{~mm}$, Fig. $4\left(f / \Delta_{S}=0.81\right.$ and $\left.x_{m}=0.74 f\right)$ gives a loss of 3.5 $\mathrm{dB}$, excluding the reflection loss. The surface wave reflection coefficient at a $90^{\circ}$ edge of aluminum is 0.40 [25], causing a loss of $8 \mathrm{~dB}$, making the total loss $11.5 \mathrm{~dB}$. This value compares very well with the experimental measurement of $11.4 \mathrm{~dB}$.

In the experiments the needed parabolic surface was approximated by a circular surface. This approximation produces negligible aberrations if the extent of the mirror is limited appropriately. On the other hand, the limited aperture of the mirror will cause degradation in the lateral resolution. The mirror can be designed such that the performance of the system is optimized to obtain a maximum amount of signal level and minimum aberration while maintaining the best possible resolution. The value of $f_{\text {ept }}$ depends upon the Schoch displacement of the material under investigation. The variation of $f_{\text {opt }}$ normalized to $\Delta_{S}$ is depicted in Fig. 16 as a function of $\Delta_{S} / \lambda$. It can be observed that the dependence of $f_{\text {opt }} / \Delta_{S}$ on material properties is rather loose. One may select $f_{\text {opt }}$ as $0.59 \Delta_{S}$ without a significant loss in performance. A formula for $\Delta_{S} / \lambda$ is given by Brekovskikh [26] in terms of material parameters. Hence $f_{\text {opt }}$ can easily be determined for a given material at a given frequency. Once $f_{\mathrm{opt}}$ is determined, the maximum width of the mirror $2 x_{m}$ can be calculated by using (12). The minimum attainable $f$ number is independent of frequency of operation and is approximately given as

$$
f \text { number }=f_{\text {opt }} / 2 x_{m}=\left\{\left[2\left(f_{\text {opt }} / \Delta_{S}\right)\left(\Delta_{S} / \lambda\right) \sin \theta_{R}\right]^{1 / 4}\right\} / 4 \text {. }
$$

For example, the $f$ number for aluminum is 0.47 and for $\mathrm{Al}_{2} \mathrm{O}_{3}$ is 0.61 .

With a surface wavelength of $2.85 \mathrm{~mm}$, it was possible to resolve periodic structures with $2-\mathrm{mm}$ periodicity. This agrees very well with the theoretical expectations. With 


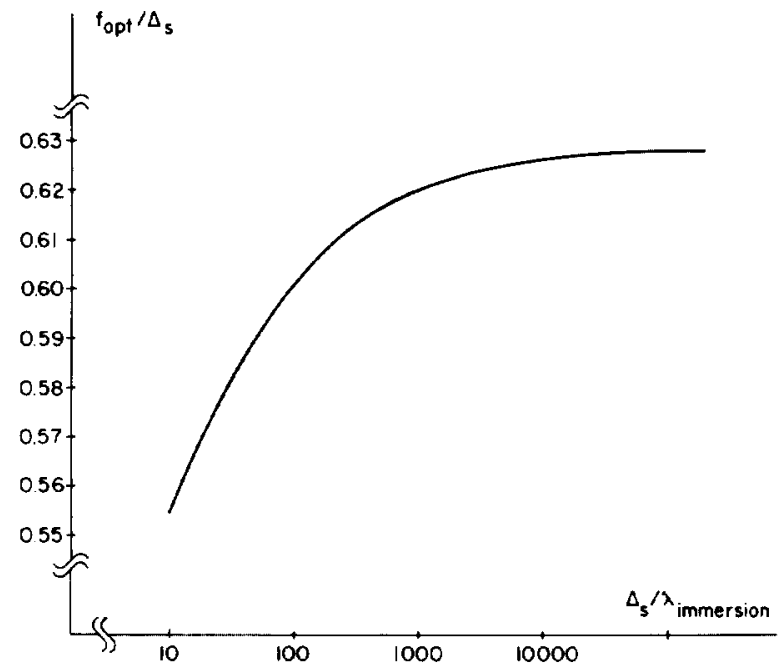

Fig. 16. Design guide to find optimum focal length for given material.

the same wavelength an inhomogeneity $2.5 \mathrm{~mm}$ below the surface was detected. Remembering that the penetration depth of a surface wave is about equal to its wavelength, this result also agrees very well with our expectations. The smallest surface hole drilled was $0.35 \mathrm{~mm}$ in diameter, and it was detected $18.5 \mathrm{~dB}$ below the edge echo level. Since there is about $35 \mathrm{~dB}$ dynamic range available in the system, small inhomogeneities or flaws much smaller than the wavelength can be detected.

Experimentation with the device has shown that it is quite easy to align the system. The alignment procedure involves positioning a single transducer at a proper angle for the excitation of surface waves for the material under investigation. There is essentially one degree of freedom in the procedure which simplifies the task considerably.

Other types of conical axicons can be used as surface wave focusing devices, provided that the angle of the cone is adjusted to equal the Rayleigh critical angle. However, a $360^{\circ}$ coverage conical beam may not be very suitable, for it receives the leaked surface waves even in the absence of the surface wave reflectors. Our partial coverage axicon is more suitable because it is basically a zero background system, generating an output signal only when a flaw is present at the surface under investigation.

A material involving layers supports many waves other than the surface waves. These waves are known as Love waves and Stonely waves. It is possible to excite these waves selectively from the liquid side if the incidence angle of the bulk wave in the liquid is properly adjusted. The resulting wave will be focused just like the surface waves. Using this mechanism, it should be possible to get much deeper penetration than is possible using surface waves which are inherently associated with the surface.

\section{ConClusion}

A new type of axicon is introduced which is very easy to fabricate and use. It is made up of a concave cylindrical surface immersed in a liquid and insonified obliquely by plane wavefronts. The waves reflected from the cylindri- cal surface form conical phase fronts. The incidence angle of the incoming waves determines the cone angle of the reflected waves. The reflected wavefronts converge into a line focus that is parallel to the axis of the cylindrical surface and at a distance equal to the half of radius of curvature.

This axicon is used to get a surface wave focusing system to be utilized in examining the plane surfaces of materials. The cone angle of the axicon is adjusted to be equal to the Rayleigh critical angle of the liquid-solid interface. The excited surface waves focus at the intersection of the material surface with the focal line of the axicon. With this simple configuration a high conversion efficiency, and therefore a high signal-to-noise ratio and sensitivity, is obtained. The resolution is limited by diffraction, and hence it is determined by the wavelength of the surface waves and the maximum convergence angle. Due to their nature the surface waves extend beneath the surface; thus the system is sensitive to variations under the surface of material. This penetration ability is limited by the wavelength of the surface waves. A lower frequency of operation will result in a better penetration ability but lower resolution performance. The system performs very close to expectations and has a promising future.

\section{ACKNOWLEDGMENT}

We thank Dr. S. Ayter for making suggestions on surface wave reflection problems.

\section{REFERENCES}

[1] H. Talaat and E. Burstein, "Phase matched electromagnetic generation and detection of surface elastic waves in nonconducting solids," J. Appl. Phys., vol. 45, pp. 4360-4362, 1972.

[2] H. L. Grubin, “Direct electromagnetic generation of compressional waves in metals in static magnetic fields," IEEE Trans. Sonics U/trason., vol. 17, pp. 227-229, 1970.

[3] 1. A. Victorov, Rayleigh and Lamb Waves. New York: Plenum Press, 1967.

[4] H. L. Bertoni and T. Tamir, "Characteristics of wedge transducers for acoustic surface waves," IEEE Trans. Sonics Ultrason., vol. SU22, pp. 415-420, 1975.

[5] J. Fraser, B. T. Khuri-Yakub, and G. S. Kino "The design of efficient broadband wedge transducers," Appl. Phys. Lett., vol. 32, pp. 698$700,1978$.

[6] B. T. Khuri-Yakub et al. , "NDE of ceramics," in Review of Progress in Quantitative NDE," vol. 1. New York: Plenum Press, 1982, pp. $601-605$.

[7] G. L. Fitzpatrick, B. P. Hildebrand, and A. J. Boland, "Acoustical imaging of near surface properties at the Rayleigh critical angle," in Acoustical Imaging vol. 12, E. A. Ash and C. R. Hill, Eds. New York: Plenum Press, 1982, pp. 157-174.

[8] I. R. Smith, H. K. Wickramasinghe, G. W. Farnell, and C. K. Jen, "Confocal surface acoustic wave microscopy," Appl. Phys. Lett., vol. 42, pp. 411-413, 1983.

[9] B. Nongaillard, M. Ourak, J. M. Rouvaen, M. Houze, and E. Bridoux, "A new focusing method for nondestructive evaluation by acoustic surface wave,"'J. Appl. Phys., vol. 55, pp. 75-79, 1984.

[10] M. S. Patterson and F. S. Foster, "Acoustic fields of conical radiators," IEEE Trans. Sonic Ultrason., vol. SU-29, pp. 83-92, 1981.

[11] G. W. Farnell and C. K. Jen, "Planar acoustic microscope lens using Rayleigh to compressional conversion," Electron. Lett., vol. 16, pp. $541-543,1980$.

[12] K. Kobayashi, T. Morizumi, and K. Toda, “Longitudinal acoustic wave radiated from an arched interdigital transducer," $J$. Appl. Phys., vol. 52, pp. 5386-5388, 1981. 
[13] R. L. Clarke, J. C. Bamber, C. R. Hill, and P. K. Wankling, "Recent developments in axicon imaging," in Acoustical Imaging, vol. 12, E. A. Ash and C. R. Hill, Eds. New York: Plenum Press, 1982, pp. $339-350$

[14] J. W. Hunt, M. Arditi, and F. S. Foster, "Ultrasonic transducers for pulse-echo medical imaging," IEEE Trans. Biomed. Eng., vol. BME30, pp. 453-481, 1983.

[15] J. H. McLeod, "The axicon: A new type of optical element," J. Opt. Soc. Amer., vol. 44, pp. 592-597, 1954.

[16] S. Fujiwara, "Optical properties of conic surfaces. 1. Reflecting cone," J. Opt. Soc. Amer., vol. 52, pp. 287-292, 1962.

[17] C. B. Burckhardt, H. Hoffmann, and P. A. Grandchamp, "Ultrasound axicon: a device for focussing over a large depth," J. Acoust. Soc. Amer., vol. 54, pp. 1628-1630, 1973

[18] O. I. Diachok and W. G. Mayer, "Conical reflection of ultrasound from a liquid-solid interface," J. Acoust. Soc. Amer., vol. 47, pp. $155-157,1970$

[19] A. N. Norris, "Back refiection of ultrasonic waves from a liquidsolid interface," J. Acoust. Soc. Amer., vol. 73, pp. 427-434, 1983.

[20] H. L. Bertoni, "Ray-optical evaluation of $V(Z)$ in the reflection acoustic microscope," IEEE Trans. Sonics Ultrason., vol. SU-31, pp. 105-116, 1984

[21] A. Atalar, "A backscattering formula for acoustic transducers," $J$. Appl. Phys., vol. S1, pp. 3093-3098, 1980.

[22] H. L. Bertoni and T. Tamir, "Unified theory of Rayleigh-angle phenomena for acoustic beams at liquid-solid interfaces," Appl. Phys., vol. 2, pp. 157-172, 1973.

[23] M. Abromowitz and I. A. Stegun, Handbook of Mathematical Functions. New York: Dover, 1970.

[24] J. W. Goodman, Fourier Optics. New York: McGraw-Hill, 1968.

[25] F. C. Cuozzo, E. L. Cambiaggio, J-P Damiano, and E. Rivier, "Influence of elastic properties on Rayleigh wave scattering by normal discontinuities," IEEE Trans. Sonics Ultrason., vol. SU-24, pp. 280 $289,1977$.

[26] L. M. Brekovskikh, Waves in Layered Media. New York: Academic, 1980.

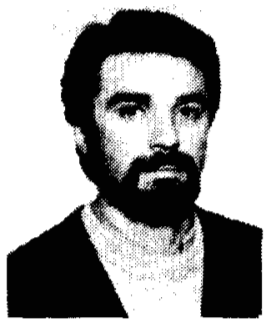

Abdullah Atalar was born in Gaziantep, Turkey, in 1954. He received the B.S. degree from Middle East Technical University, Ankara, Turkey, in 1974 , and the M.S. and Ph.D. degrees from Stanford University, Stanford, CA, in 1976 and 1978, respectively, all in electrical engineering.

From 1978 to 1980 he was first a Post Doctoral Fellow and later an Engineering Research Associate in Stanford University continuing his work on acoustic microscopy. For eight months he was with Hewlett Packard Labs. Palo Alto, CA, engaged in photoacoustics research. In 1980 he joined the Middle East Technical University as an Assistant Professor. From 1982 to 1983 on leave from University, he was with Ernst Leitz Wetzlar, Germany, involved in the development of the commercial acoustic microscope. He is presently an Associate Professor in the Electrical and Electronics Engineering Department of Middle East Technical University and a consultant for ASELSAN, Ankara. His current research interests include acoustic imaging, ljnear acoustics, and computer aided design in electrical engineering.

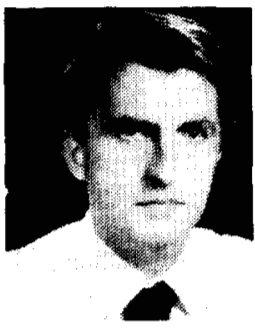

Hayrettin Köymen was born in Ankara, Turkey, on June 7, 1952. He received the B.Sc. and M.Sc. degrees from Middle East Technical University, Ankara, Turkey, in 1973 and 1976 , respectively, and Ph.D. degree from University of Birmingham, England, in 1979, all in electrical engineering.

He joined the Middle East Technical University in 1979 as an Assistant Professor, where he first worked on underwater acoustics and oceanographic instrumentation until 1982, and on medical ultrasonic imaging and ultrasonic NDE afterwards. His current research interests are ultrasonic transducers, quantitative medical ultrasonic imaging, and imaging for NDE. 